International Journal of Pure and Applied Mathematics

Volume 85 No. 3 2013, 495-506

ISSN: 1311-8080 (printed version); ISSN: 1314-3395 (on-line version)

url: http://www.ijpam.eu

doi: http://dx.doi.org/10.12732/ijpam.v85i3.6

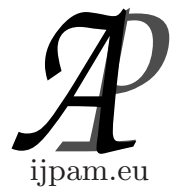

\title{
NUMERICAL SOLUTION OF LINEAR DIRICHLET TWO-POINT BOUNDARY VALUE PROBLEMS USING BLOCK METHOD
}

\author{
Mohd Mughti Hasni ${ }^{1}$, Zanariah Abdul Majid ${ }^{2}$, Norazak Senu ${ }^{3}$ \\ ${ }^{1,2,3}$ Institute for Mathematical Research \\ Universiti Putra Malaysia \\ 43400 UPM, Serdang, Selangor, MALAYSIA
}

\begin{abstract}
This paper presents a direct two-point block one-step method for solving linear Dirichlet boundary value problems (BVPs) directly. The block method is formulated using Lagrange interpolating polynomial. Mathematical problems which involve higher order ordinary differential equations (ODEs) were likely to be reduced into the system of first order equations in order to solve it. However, this method will solve the second order linear Dirichlet BVPs directly without reducing it to the system of first order equations. The direct solution of the linear Dirichlet BVPs will be calculated at the two-points simultaneously using constant step size. This method will be used together with the linear shooting technique to construct the numerical solution. The implementation is based on the predictor and corrector formulas in the $\mathrm{PE}(\mathrm{CE})^{r}$ mode. Numerical results are given to show the efficiency and performance of this method compared to the existing methods.
\end{abstract}

AMS Subject Classification: 65L06, 65L10

Key Words: linear Dirichlet boundary value problems, block method, linear shooting method, constant step size

\section{Introduction}

Boundary Value Problems (BVPs) is a differential equation with a set of addi-

Received: January 24, 2013

(C) 2013 Academic Publications, Ltd.

${ }^{\S}$ Correspondence author url: www.acadpubl.eu 
tional restraints, called a boundary conditions. The BVPs solution is a solution of differential equation which also satisfies the boundary conditions. BVPs play an important role in many fields such as physics, chemistry and engineering. The two-point BVPs occur in a variety of problems, including the modeling of chemical reaction, heat transfer, diffusion, and the solution of optimal control problems. There are fewer types of BVPs and some of them depend on the boundary conditions itself.

In this paper, we consider the second order linear two-point BVPs which as follows:

$$
y^{\prime \prime}=p(x) y^{\prime}+q(x) y+r(x), q(x)>0, a \leq x \leq b
$$

with the Dirichlet boundary conditions,

$$
y(a)=\alpha, y(b)=\beta .
$$

There are many techniques available for the numerical solution of two-point BVPs such as the extended Adomian decomposition in [1], the extended cubic B-spline in [5] and the combined homotophy perturbation with Green's function methods in [10].

In 2002, Fang et al. [3] have applied the finite difference, the finite element and the finite volume methods to obtain the solution for the two-point BVPs. The comparison has been made for this three difference types of method and the approximated results show that they are comparable to each other with no remarkable differences. Later in 2006, Caglar et al. [2] have improved the approximated results compared with Fang et al. [3] by using the B-spline interpolation. By applying the same procedure as the B-spline interpolation, Hamid et al. [5] have proposed a new method called the extended cubic B-spline for solving linear two-point BVPs. This method solved the BVPs by transforming it into the piecewise polynomial function. The approximated results have improved by using only five or six iterations for the method to converge. The Adomian decomposition method has been widely studied by many researchers in solving differential and integral problems in many scientific applications. This method decomposed the solutions into the series which converge rapidly. Unfortunately, this method is not suitable to solve BVPs. Therefore, based on this method, Bongsoo [1] have introduced a new method called the extended Adomian decomposition method for solving linear and non-linear two-point BVPs.

Several researchers such as Fatunla [4], Majid et al. [6], Rosser [8] and Shampine and Watts [9] have proposed a block method which computes concurrently the solution values at different points along the interval. Recently in 
2011, Majid et al. [7] have derived the direct two-point block one-step method for solving general second order ODEs directly without reducing it into the system of first order equations. The result shown that it was better in terms of the accuracy and the computed times. Thus, we are motivated to implement this method for solving the linear Dirichlet BVPs.

In this research, we have extend the idea in [7] for solving (1) directly without reducing it into the system of first-order using the direct two-point block one-step method with the linear shooting techniques.

\section{Formulation}

From Figure 1, based in Majid et al.[7], the method will compute the two approximation values $y_{n+1}$ at $x_{n+1}$ and $y_{n+2}$ at $x_{n+2}$ simultaneously in the block.

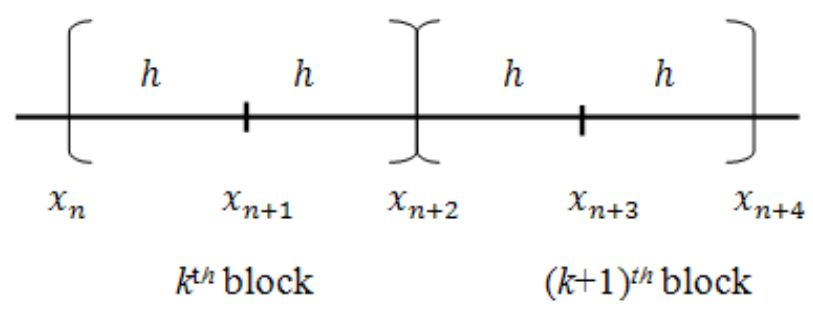

Figure 1: Two-Point Block One-Step Method

The interval of $[a, b]$ is divided into a series of block with each block containing two-points with the step size $2 h$. Then, the solution obtained in the last point within the $\mathrm{k}^{\text {th }}$ block will be restored as the initial value for the next block. The same procedure will be used to compute the solutions until the end of the interval. This method possesses the desirable feature of one-step method in which being referred to only one previous point to obtain the solution.

Now, by letting the second order differential equation as follows:

$$
y^{\prime \prime}=f\left(x, y, y^{\prime}\right)
$$

The approximating values of $y_{n+1}$ and $y_{n+2}$ was obtained by integrating once and twice over (2) with respect to $x$. We start to evaluate the first point $y_{n+1}$ and $y_{n+1}^{\prime}$ by integrating (2) once and twice both left and the right hand side 
over the interval as follows:

$$
\begin{aligned}
\int_{x_{\mathrm{n}}}^{x_{\mathrm{n}+1}} y^{\prime \prime}(x) d x & =\int_{x_{\mathrm{n}}}^{x_{\mathrm{n}+1}} f\left(x, y, y^{\prime}\right) d x \\
\int_{x_{\mathrm{n}}}^{x_{\mathrm{n}+1}} \int_{x_{\mathrm{n}}}^{x} y^{\prime \prime}(x) d x d x & =\int_{x_{\mathrm{n}}}^{x_{\mathrm{n}+1}} \int_{x_{\mathrm{n}}}^{x} f\left(x, y, y^{\prime}\right) d x d x
\end{aligned}
$$

Therefore,

$$
\begin{aligned}
y^{\prime}\left(x_{n+1}\right)-y^{\prime}\left(x_{n}\right) & =\int_{x_{\mathrm{n}}}^{x_{\mathrm{n}+1}} f\left(x, y, y^{\prime}\right) d x \\
y\left(x_{n+1}\right)-y\left(x_{n}\right)-h y^{\prime}\left(x_{n}\right) & =\int_{x_{\mathrm{n}}}^{x_{\mathrm{n}+1}}\left(x_{n+1}-x\right) f\left(x, y, y^{\prime}\right) d x
\end{aligned}
$$

Thus, Lagrange interpolating polynomial will replace the $f\left(x, y, y^{\prime}\right)$ in (4). The interpolation points involved the points $\left(x_{n}, f_{n}\right),\left(x_{n+1}, f_{n+1}\right)$ and $\left(x_{n+2}, f_{n+2}\right)$ within the block. Now, by taking $x=x_{n+2}+s h ; d x=h d s$ and replacing into (4) and then taking -2 to -1 as the limit of integration. Hence, the corrector formulae for the first point will be obtained as follows:

$$
\begin{aligned}
& y_{n+1}^{\prime}=y_{n}^{\prime}+\frac{h}{12}\left(5 f_{n}+8 f_{n+1}-f_{n+2}\right) \\
& y_{n+1}=y_{n}+h y_{n}^{\prime}+\frac{h^{2}}{24}\left(7 f_{n}+6 f_{n+1}-f_{n+2}\right)
\end{aligned}
$$

To obtain the corrector formula for the second point in which the approximate value of $y_{n+2}^{\prime}$ and $y_{n+2}$ are needed. The same process as above will be repeated as to obtain the corrector formulae for the first point except by integrating it over the interval $\left[x_{n+1}, x_{n+2}\right]$.

$$
\begin{aligned}
\int_{x_{\mathrm{n}+1}}^{x_{\mathrm{n}+2}} y^{\prime \prime}(x) d x & =\int_{x_{\mathrm{n}+1}}^{x_{\mathrm{n}+2}} f\left(x, y, y^{\prime}\right) d x \\
\int_{x_{\mathrm{n}+1}}^{x_{\mathrm{n}+2}} \int_{x_{\mathrm{n}+1}}^{x} y^{\prime \prime}(x) d x d x & =\int_{x_{\mathrm{n}+1}}^{x_{\mathrm{n}+2}} \int_{x_{\mathrm{n}+1}}^{x} f\left(x, y, y^{\prime}\right) d x d x
\end{aligned}
$$

Therefore,

$$
\begin{aligned}
y^{\prime}\left(x_{n+2}\right)-y^{\prime}\left(x_{n+1}\right) & =\int_{x_{n+1}}^{x_{n+2}} f\left(x, y, y^{\prime}\right) d x \\
y\left(x_{n+2}\right)-y\left(x_{n+1}\right)-h y^{\prime}\left(x_{n+1}\right) & =\int_{x_{n+1}}^{x_{n+2}}\left(x_{n+2}-x\right) f\left(x, y, y^{\prime}\right) d x
\end{aligned}
$$


Now, the limit of integration would be from -1 to 0 and the corrector formulae would be as follows

$$
\begin{aligned}
& y_{n+2}^{\prime}=y_{n+1}^{\prime}+\frac{h}{12}\left(-f_{n}+8 f_{n+1}+5 f_{n+2}\right) \\
& y_{n+2}=y_{n+1}+h y_{n+1}^{\prime}+\frac{h^{2}}{24}\left(-f_{n}+10 f_{n+1}+3 f_{n+2}\right)
\end{aligned}
$$

Based on Fatunla [4], the method is said to be of order $p$ if $C_{0}=C_{1}=C_{2}=$ $\ldots=C_{p+1}=0$ and $C_{p+2} \neq 0$ is the error constant. Then, we can see that the methods in (5) and (8) are of the order 3 and the error constant as follows:

$$
C_{5}=C_{p+2}=\left[\frac{1}{24}, \frac{1}{45}, \frac{-1}{24} \frac{-7}{36}\right]^{T}
$$

For detail, please refer to Majid et al. [7].

\section{Implementation}

The Euler method will be used as the initial starting point for each block as a predictor. This method will be based on the predictor and corrector formulas with the linear shooting in the $P E(C E)^{r}$ mode where $P$ and $C$ denote the application of predictor and corrector respectively while $E$ denote the evaluation of function $f$ and the $r$ indicates the number of iteration that is needed. For each step, we use $r$ functions evaluations until the convergence is satisfied. Since this method will be based on the predictor and corrector formulae $P E(C E)^{r}$ mode, we will correct the approximate solution obtained from the predictor with the corrector formulae. For the next block, the same process will be applied to compute the approximation values of $y_{n+1}$ and $y_{n+2}$ concurrently up to the end of the interval.

The idea to solve the BVPs was by combining together this two-point block one-step method with the linear shooting technique. The BVPs (1) will be replaced by the two initial value problems (IVP) as follows:

$$
\begin{array}{ll}
y_{1}^{\prime \prime}=p(x) y_{1}^{\prime}+q(x) y_{1}+r(x), & y_{1}(a)=\alpha, y_{1}^{\prime}(a)=0 \\
y_{2}^{\prime \prime}=p(x) y_{2}^{\prime}+q(x) y_{2}, & y_{2}(a)=0, y_{2}^{\prime}(a)=1
\end{array}
$$

The linear shooting method was obtained by performing the linear combination between the nonhomogeneous and homogeneous equations in (10). Hence, a 
new function that is linear shooting method will be constructed by solving the two IVPs:

$$
y(x)=y_{1}(x)+w y_{2}(x)
$$

with

$$
w=\frac{\beta-y_{1}(b)}{y_{2}(b)}
$$

This value of $w$ were obtained from the substitution of the last point in the interval into the linear shooting method (11) giving us:

$$
y(b)=y_{1}(b)+w y_{2}(b)
$$

The code will be implemented using constant step size $h$. The convergence test will be used during the calculation of the approximated solution in the corrector formulae. The convergence test is as follows:

$$
\left|y_{2, r+1}-y_{2, r}\right|<0.1 \times \mathrm{TOL}
$$

where $r$ is the number of iterations and TOL is the tolerance. In the code we control the convergent test at the second point. All problems were tested using the absolute error test. The iterations in the corrector formulae will be repeated until the convergence test was satisfied. In order for us to obtained the best result for this method, TOL $=10^{-5}$ were used for the step size 0.1 and 0.125. While $\mathrm{TOL}=10^{-8}$ was used for the step size 0.01 .

\section{Numerical Results}

We would like to demonstrate the effectiveness of this method towards this three tested problems. The comparison has been made by showing the local and maximum error obtained by the direct two-point block one-step method with the existing methods.

$$
\text { Problem 1. } \frac{d^{2} y}{d x^{2}}=y+\cos (x),[0,1] \text {. }
$$

Boundary condition: $y(0)=0, y(1)=1$.

Exact solution:

$$
\begin{aligned}
y(x)= & \frac{-3 \cosh (1)+3 \sinh (1)+\cos (1)+2}{4 \sinh (1)} e^{x} \\
& +\frac{3 \cosh (1)+3 \sinh (1)-\cos (1)-2}{4 \sinh (1)} e^{-x}-\frac{\cos x}{2} .
\end{aligned}
$$

Source: Bongsoo [1] 
Problem 2:

$\frac{d^{2} y}{d x^{2}}=\frac{d y}{d x}-\exp (x-1)-1,[0,1]$.

Boundary condition: $y(0)=0, y(1)=0$.

Exact solution:

$y(x)=x(1-\exp (x-1))$.

Source: Hamid et al. [5]

Problem 3:

$\frac{d^{2} y}{d x^{2}}=-(x+1) \frac{d y}{d x}+2+\left(1-x^{2}\right)-e^{-x},[0,1]$.

Boundary condition: $y(0)=-1, y(1)=0$.

Exact solution:

$y(x)=(x-1) e^{-x}$.

Source: Hamid et al. [5].

\section{Notations}

MAXE : Maximum Error of the Computed Solution

TS : Total steps taken

$\operatorname{ECBIM}(\mathrm{N})$ : Extended Cubic B-Spline Method Minimizing Using

Newton's Method in Hamid et al. [5]

ECBIM(B) : Extended Cubic B-Spline Method Minimizing Using

Built-In Function in Hamid et al. [5]

EADM : Extended Adomian Decomposition Method in Bongsoo

[1]

2PLBVP : Implementation Of The Direct Two-Point Block One-

Step Method For Solving the Linear Dirichlet BVPs

* $\quad$ : Maximum Error of the Computed Solution

In Table 1, the approximated solutions for the 2PLBVP were better than the EADM. The comparison between the maximum errors for both of this method clearly indicate that the 2PLBVP can give better accuracy despite the advantages with this methods itself which solved the problem directly without reducing it into the system of first order equations. The 2PLBVP manage to approximate the solutions at two points simultaneously along the interval, 


\begin{tabular}{|c|c|c|c|c|}
\hline$x$ & $\begin{array}{l}\text { MAXE } \\
\text { EADM }\end{array}$ & $\begin{array}{c}\text { TS } \\
\text { EADM }\end{array}$ & $\begin{array}{c}\text { MAXE } \\
\text { 2PLBVP }\end{array}$ & $\begin{array}{c}\text { TS } \\
\text { 2PLBVP }\end{array}$ \\
\hline $1 / 8$ & $4.37 \times 10^{-7}$ & \multirow{7}{*}{8} & $1.47 \times 10^{-7}$ & \multirow{7}{*}{4} \\
\hline $2 / 8$ & $8.07 \times 10^{-7}$ & & $3.24 \times 10^{-7}$ & \\
\hline $3 / 8$ & $1.05 \times 10^{-6}$ & & $3.68 \times 10^{-7}$ & \\
\hline $4 / 8$ & $1.14 \times 10^{-6}$ & & $4.30 \times 10^{-7}$ & \\
\hline $5 / 8$ & $1.05 \times 10^{-6}$ & & $3.60 \times 10^{-7}$ & \\
\hline $6 / 8$ & $8.07 \times 10^{-7}$ & & $3.07 \times 10^{-7}$ & \\
\hline $7 / 8$ & $4.37 \times 10^{-7}$ & & $1.41 \times 10^{-7}$ & \\
\hline
\end{tabular}

Table 1: Numerical result for solving Problem 1 when $h=0.125$

\begin{tabular}{|c|c|c|c|c|c|}
\hline $\begin{array}{l}\text { Existing Method } \\
\qquad(h=0.1)\end{array}$ & $\mathrm{TS}$ & MAXE & $\begin{array}{c}x \\
(\mathrm{~h}=0.1)\end{array}$ & TS & $\begin{array}{c}\text { MAXE } \\
2 \mathrm{PLBVP}\end{array}$ \\
\hline \multirow[t]{7}{*}{$\operatorname{ECBIM}(\mathrm{N})$} & \multirow[t]{7}{*}{10} & \multirow[t]{7}{*}{$7.91 \times 10^{-6}$} & 0.0 & \multirow{11}{*}{5} & - \\
\hline & & & 0.1 & & $2.71 \times 10^{-7}$ \\
\hline & & & 0.2 & & $5.26 \times 10^{-7}$ \\
\hline & & & 0.3 & & $6.95 \times 10^{-7}$ \\
\hline & & & 0.4 & & $8.57 \times 10^{-7}$ \\
\hline & & & 0.5 & & $9.21 \times 10^{-7}$ \\
\hline & & & 0.6 & & $9.77 \times 10^{-7 *}$ \\
\hline \multirow[t]{4}{*}{$\operatorname{ECBIM}(\mathrm{B})$} & \multirow[t]{4}{*}{10} & \multirow[t]{4}{*}{$5.73 \times 10^{-6}$} & 0.7 & & $9.00 \times 10^{-7}$ \\
\hline & & & 0.8 & & $7.90 \times 10^{-7}$ \\
\hline & & & 0.9 & & $4.60 \times 10^{-7}$ \\
\hline & & & 1.0 & & $1.11 \times 10^{-16}$ \\
\hline
\end{tabular}

Table 2: Numerical result for solving Problem 2 when $h=0.1$

hence the total steps will be half compared to EADM.

Table 2 and Table 3 gave the approximate numerical results for problem 2 based on their step size $h=0.1$ and $h=0.01$ respectively. The maximum error 


\begin{tabular}{|c|c|c|c|c|c|}
\hline $\begin{array}{l}\text { Existing Method } \\
\quad(h=0.01)\end{array}$ & $\mathrm{TS}$ & MAXE & $\begin{array}{c}x \\
(\mathrm{~h}=0.01)\end{array}$ & $\mathrm{TS}$ & $\begin{array}{c}\text { MAXE } \\
2 \mathrm{PLBVP}\end{array}$ \\
\hline \multirow[t]{11}{*}{ EADM } & \multirow[t]{11}{*}{100} & \multirow[t]{11}{*}{$1.05 \times 10^{-10}$} & 0.0 & \multirow[t]{11}{*}{50} & - \\
\hline & & & 0.1 & & $9.21 \times 10^{-11}$ \\
\hline & & & 0.2 & & $1.68 \times 10^{-10}$ \\
\hline & & & 0.3 & & $2.29 \times 10^{-10}$ \\
\hline & & & 0.4 & & $2.75 \times 10^{-10}$ \\
\hline & & & 0.5 & & $3.04 \times 10^{-10}$ \\
\hline & & & 0.6 & & $3.15 \times 10^{-10 *}$ \\
\hline & & & 0.7 & & $3.01 \times 10^{-10}$ \\
\hline & & & 0.8 & & $2.56 \times 10^{-10}$ \\
\hline & & & 0.9 & & $1.64 \times 10^{-10}$ \\
\hline & & & 1.0 & & $6.66 \times 10^{-16}$ \\
\hline
\end{tabular}

Table 3: Numerical result for solving Problem 2 when $h=0.01$

in Table 2 clearly shows that the 2PLBVP gave better result when $h=0.1$ compared with both results in $\operatorname{ECBIM}(\mathrm{N})$ and ECBIM (B). For the step size $h=0.01$ in Table $3,2 \mathrm{PLBVP}$ gave a comparable result with the EADM and the total steps are lesser compared to EADM.

In Table 4, the approximated solution of problem 3 were more precise compared to the existing methods which is ECBIM (N) and ECBIM (B). Meanwhile, Table 5 give the local errors at each step for problem 3 with step size $h=0.01$ and total steps $=50$. The resulting results indicate that the $2 \mathrm{PLBVP}$ can give better accuracy as the step size decreased.

\section{Conclusion}

In this research, we have implemented the direct two-point block one-step method together with the linear shooting technique with constant step size which is suitable for solving the second order linear Dirichlet BVPs directly. This method has shown the acceptable solution based on the tested problems. 


\begin{tabular}{|c|c|c|c|c|c|}
\hline $\begin{array}{l}\text { Existing Method } \\
\quad(h=0.1)\end{array}$ & $\mathrm{TS}$ & MAXE & $\begin{array}{c}x \\
(\mathrm{~h}=0.1)\end{array}$ & TS & $\begin{array}{c}\text { MAXE } \\
2 \mathrm{PLBVP}\end{array}$ \\
\hline \multirow[t]{6}{*}{$\operatorname{ECBIM}(\mathrm{N})[6]$} & \multirow[t]{6}{*}{10} & \multirow[t]{6}{*}{$6.61 \times 10^{-6}$} & 0.0 & \multirow{11}{*}{5} & - \\
\hline & & & 0.1 & & $1.13 \times 10^{-7}$ \\
\hline & & & 0.2 & & $2.21 \times 10^{-7}$ \\
\hline & & & 0.3 & & $2.40 \times 10^{-7}$ \\
\hline & & & 0.4 & & $2.63 \times 10^{-7 *}$ \\
\hline & & & 0.5 & & $2.31 \times 10^{-7}$ \\
\hline \multirow[t]{5}{*}{$\operatorname{ECBIM}(\mathrm{B})[6]$} & \multirow[t]{5}{*}{10} & \multirow[t]{5}{*}{$4.97 \times 10^{-6}$} & 0.6 & & $2.12 \times 10^{-7}$ \\
\hline & & & 0.7 & & $1.65 \times 10^{-7}$ \\
\hline & & & 0.8 & & $1.18 \times 10^{-7}$ \\
\hline & & & 0.9 & & $4.27 \times 10^{-8}$ \\
\hline & & & 1.0 & & $4.08 \times 10^{-17}$ \\
\hline
\end{tabular}

Table 4: Numerical result for solving Problem 3 when $h=0.1$

\begin{tabular}{|c|c|c|}
\hline$x$ & $\begin{array}{c}\text { 2PLBVP } \\
h=0.01\end{array}$ & \multirow{2}{*}{$\begin{array}{c}\text { TS } \\
\text { 2PLBVP }\end{array}$} \\
\hline 0.0 & - & \\
\hline 0.1 & $4.229710^{-10}$ & \multirow{2}{*}{50} \\
\hline 0.2 & $7.652410^{-10}$ & \\
\hline 0.3 & $9.345910^{-10 *}$ & \\
\hline 0.4 & $8.787710^{-10}$ \\
\hline 0.5 & $6.105310^{-10}$ \\
\hline 0.6 & $2.246910^{-10}$ \\
\hline 0.7 & $9.723110^{-11}$ \\
\hline 0.8 & $1.050610^{-11}$ \\
\hline 0.9 & $4.338910^{-12}$ \\
\hline 1.0 & $2.450610^{-16}$ \\
\hline
\end{tabular}

Table 5: Numerical result for solving Problem 3 when $h=0.01$ 


\section{Acknowledgments}

We would like to thank Ministry of Higher Education Malaysia for providing financial support through Fundamental Research Grant Scheme (FRGS) during the study period.

\section{References}

[1] J. Bongsoo, Two-point boundary value problems by the extended Adomian decomposition method, Journal of Computational and Applied Mathematics , 219, No. 1 (2008), 253-262.

[2] H. Caglar, N. Caglar, K.El Faituri, B-spline interpolation compared with finite difference, finite element and finite volume methods which applied to two-point boundary value problems, Applied Mathematics and Computation, 175 (2006), 72-79.

[3] Q. Fang, T. Tsuchiya, T. Yamamoto, Finite difference, finite element and finite volume methods applied to two-point boundary value problems, Journal of Computational and Applied Mathematics, 139, No. 1 (2002), 9-19.

[4] S.O. Fatunla, Block methods for second order ODEs, International Journal of Computer Mathematics, 41 (1991), 55-63.

[5] N.N.A. Hamid, A.A. Majid, A.I.M. Ismail, Extended cubic B-spline method for linear two-point boundary value problems, Sains Malaysiana, 40, No. 11 (2011), 1285-1290.

[6] Z.A. Majid, P.S. Phang, M. Suleiman, Application of block method for solving nonlinear two-point boundary value problem, Advance Science Letter, 13 (2012), 754-757.

[7] Z.A. Majid, N.Z. Mokhtar, M. Suleiman,Direct two-point block one-step method for solving general second-order ordinary differential equations, Mathematical Problems in Engineering, 2012, Article ID 184253, (2011) 16 pages, doi: $10.1155 / 2012 / 184253$

[8] J.B. Rosser, A Runge-Kutta for all seasons, SIAM Review, 9 (1967) 417452. 
[9] L.F. Shampine, H.A. Watts, Block implicit one-step methods, Mathematics of Computation, 23 (1969), 731-740.

[10] Y.G. Wang, H.F. Song, D. Li, Solving two-point boundary value problems using combined homotopy perturbation method and Green's function method, Applied Mathematics and Computation, 212, No. 2 (2009), 366-376. 